\title{
Refractive-index measurement of absorbing condensed media
}

\author{
W. Leupacher and A. Penzkofer
}

\begin{abstract}
An experimental arrangement is described for determining refractive indices of absorbing liquids and solids over a wide spectral range. Reflection and transmission measurements are carried out. Refractive indices of rhodamine $6 \mathrm{G}$-methanol solutions around the $S_{1}$ absorption band are presented.
\end{abstract}

\section{Introduction}

The refractive index of nonabsorbing or weakly $a b$ sorbing liquids and solids is generally measured by refraction or total internal reflection in prisms. ${ }^{1-3}$ An accuracy of $\Delta n= \pm 10^{-4}$ is achieved. With increasing absorption the prism techniques become more and more inaccurate; small apex angles have to be used because of reduced transmission. The real angle of refraction $\chi_{t}$ is related in a complicated way to the complex angle of refraction $\chi$ obtained from Snell's law. $4-7$

Reflection techniques (measurement of reflected light intensity) or ellipsometric techniques (measurement of amplitude and phase of reflected electrical field strength) are applied to absorbing media. ${ }^{8-10}$ The reflection of light at a plane interface between two media depends on the angle of incidence, the light polarization, and the refractive indices as well as the absorption coefficients of the adjoining media. For moderate absorption (absorption coefficient $\alpha<7000 \mathrm{~cm}^{-1}$, extinction coefficient $k<0.03$, see below) the absorption parameter is measurable separately by transmission measurements, and the refractive index may be determined by detecting the reflection of light at a fixed angle of incidence $\varphi$ near $0^{\circ} .11,12$ For strongly absorbing substances both optical constants, refractive index $n$ and extinction coefficient $k$, have to be deduced from ellipsometric ${ }^{13-16}$ or reflection measurements ${ }^{17-26}$ (use of different polarizations, ${ }^{17-24}$ different angles of incidence, ${ }^{17-24}$ modulation of polarization, ${ }^{24}$ modulation of angle of incidence, ${ }^{13-16}$ Kramers-Kronig integras tion $^{26}$ ).

\footnotetext{
The authors are with Universität Regensburg, Naturwissenschaf tliche Fakultat II-Physik, 8400 Regensburg, Federal Republic of Germany.

Received 7 January 1984.

0003-6935/84/101554-05\$02.00/0.

(c) 1984 Optical Society of America.
}

In this paper we describe an experimental system to determine refractive indices of liquids and solids over a wide frequency region (near UV to near IR) by reflection measurement at a small angle of incidence and separate absorption detection. The accuracy of the technique is analyzed. As an example, refractive-index data of rhodamine 6G dissolved in methanol are presented in the wavelength region between 400 and 600 $\mathrm{nm}$. Refractive indices of organic dye solutions have been needed for the calculation of nonlinear optical susceptibilities from nonphase matched third harmonic generation. 27,28

\section{Theory}

The reflection of light at the interface between air (nonabsorbing) and liquid or solid is illustrated in Fig. 1. The angles of incidence $\varphi$ and refraction $\chi_{t}$ as well as the electrical field strengths $E_{\|}$(polarized parallel to the plane of incidence) and $E_{\perp}$ are indicated. The reflection is determined by the Fresnel laws ${ }^{4-6}$ :

$$
\begin{aligned}
\frac{E_{r \perp}}{E_{i \perp}} & =\frac{\cos \varphi-n^{\prime} \mu \cos \chi}{\cos \varphi+n^{\prime} \mu \cos \chi}=\frac{\cos \varphi-\mu\left(n^{\prime 2}-\sin ^{2} \varphi\right)^{1 / 2}}{\cos \varphi+\mu\left(n^{\prime 2}-\sin ^{2} \varphi\right)^{1 / 2}}, \\
\frac{E_{r \|}}{E_{i \|}} & =\frac{n^{\prime} \mu \cos \varphi-\cos \chi}{n^{\prime} \mu \cos \varphi-\cos \chi}=\frac{n^{\prime 2} \mu \cos \varphi-\left(n^{\prime 2}-\sin ^{2} \varphi\right)^{1 / 2}}{n^{\prime 2} \mu \cos \varphi+\left(n^{\prime 2}-\sin ^{2} \varphi\right)^{1 / 2}} .
\end{aligned}
$$

The second part of the equations is obtained by use of the generalized Snell's law:

$$
\sin \varphi=n^{\prime} \sin \chi,
$$

$\chi$ is the complex refraction angle. $n^{\prime}=n_{t}^{\prime} / n_{i}=\left(n_{t}-\right.$ $\left.i k_{t}\right) / n_{i}=n-i k$ is the relative complex refractive index. $n=n_{t} / n_{i}$ is the real relative refractive index, while the absorption is given by $k=k_{t} / n_{i}\left(n_{i} \simeq 1.00027\right.$ in case of $\left.\operatorname{air}^{3}\right) . \quad k_{t}=\alpha_{t} /(4 \pi \tilde{\nu})$ is the extinction coefficient of the medium at frequency $\nu=c \tilde{\nu} . \quad \alpha_{t}=-\ln (T) / l$ is the absorption coefficient, while $T$ is the pure transmission through the substance and $l$ is the sample length. The ratio $\mu=\mu_{i} / \mu_{t}$ is the ratio of the relative permeabilities. For dielectrics $\mu_{i}$ and $\mu_{t}$ are very close to $1, s 0 \mu=1$ is used in the figures and the experimental analysis presented. 


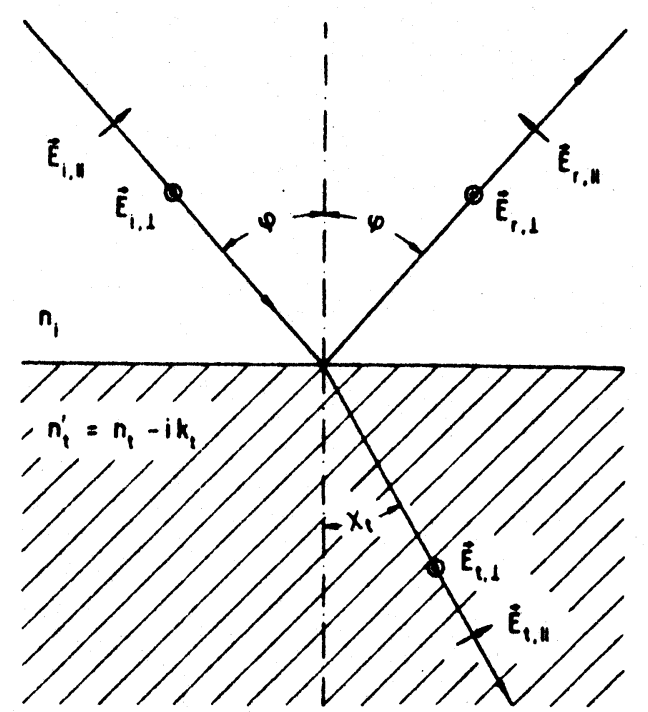

Fig. 1. Reflection and refraction at a plane interface.

The reflectances $R=\left|E_{r} / E_{i}\right|^{2}$ are readily derived from Eqs. (1) and (2). The result is

$$
\begin{aligned}
R_{\perp} & =\frac{\cos ^{2} \varphi+\mu^{2} A-2 \mu A^{1 / 2} \cos \varphi \cos B}{\cos ^{2} \varphi+\mu^{2} A+2 \mu A^{1 / 2} \cos \varphi \cos B} \\
R_{\|} & =\frac{\mu^{2}\left(C^{2}+D^{2}\right) \cos ^{2} \varphi+A-2 \mu A^{1 / 2} \cos \varphi(C \cos B+D \sin B)}{\mu^{2}\left(C^{2}+D^{2}\right) \cos ^{2} \varphi+A+2 \mu A^{1 / 2} \cos \varphi(C \cos B+D \sin B)}
\end{aligned}
$$

with $A=\left[\left(n^{2}-k^{2}-\sin ^{2} \varphi\right)^{2}+4 n^{2} k^{2}\right]^{1 / 2}, B=0.5$ arc$\tan \left[2 n k /\left(n^{2}-k^{2}-\sin ^{2} \varphi\right)\right], C=n^{2}-k^{2}$, and $D=$ $2 n k$.

In the experiments unpolarized incident light is used and the reflectivity is

$$
R_{u}=\frac{1}{2}\left(R_{\perp}+R_{\|}\right)
$$

Direct analytical solutions for $n$ and $k$ from Fresnel Eqs. (1) and (2) are given in Ref. 29. Here Eqs. (4) and (5) are solved numerically by a trial and error method.

For normal incidence $\varphi=0$, Eqs. (4) and (5) simplify to

$$
R=R_{\|}=R_{\perp}=R_{u}=\left|\frac{1-\mu n^{\prime}}{1+\mu n^{\prime}}\right|^{2}=\frac{(1-\mu n)^{2}+\mu^{2} k^{2}}{(1+\mu n)^{2}+\mu^{2} k^{2}},
$$

or

$$
n=\frac{1}{\mu}\left\{\frac{1+R}{1-R} \pm\left[\left(\frac{1+R}{1-R}\right)^{2}-1-\mu^{2} k^{2}\right]^{1 / 2}\right\} .
$$

As an illustration, in Fig. 2 the reflectances $R_{\|}, R_{u}$, and $R_{\perp}$ are plotted vs $\varphi$ for $n=1.5, k=0$ (solid curves), $n$ $=1.5, k=1$ (dashed curves), and $n=2.42, k=0$ (dash-dot curves). $R_{u}$ is practically constant up to an angle of $\varphi=20^{\circ}$. In our experiments the angle of incidence is $\varphi=6.8^{\circ}$, and it is found that $\mid n(\varphi=0)-n(\varphi$ $\left.=6.8^{\circ}\right) \mid<5 \times 10^{-5}$ for $0<R_{u}<0.5$. Therefore Eq. (8) is used in the experiments to calculate $n$ (unpolarized incident light). It should be noted that when $k>0$, no Brewster angle $\varphi_{B}$ with $R_{\|}\left(\varphi_{B}\right)=0$ exists. ${ }^{30,31}$ The $R_{\perp}$ and $R_{u}$ curves for $n=1.5, k=1$ and $n=2.42, k=0$ (same reflectance at $\varphi=0^{\circ}$ ) differ only slightly indi-

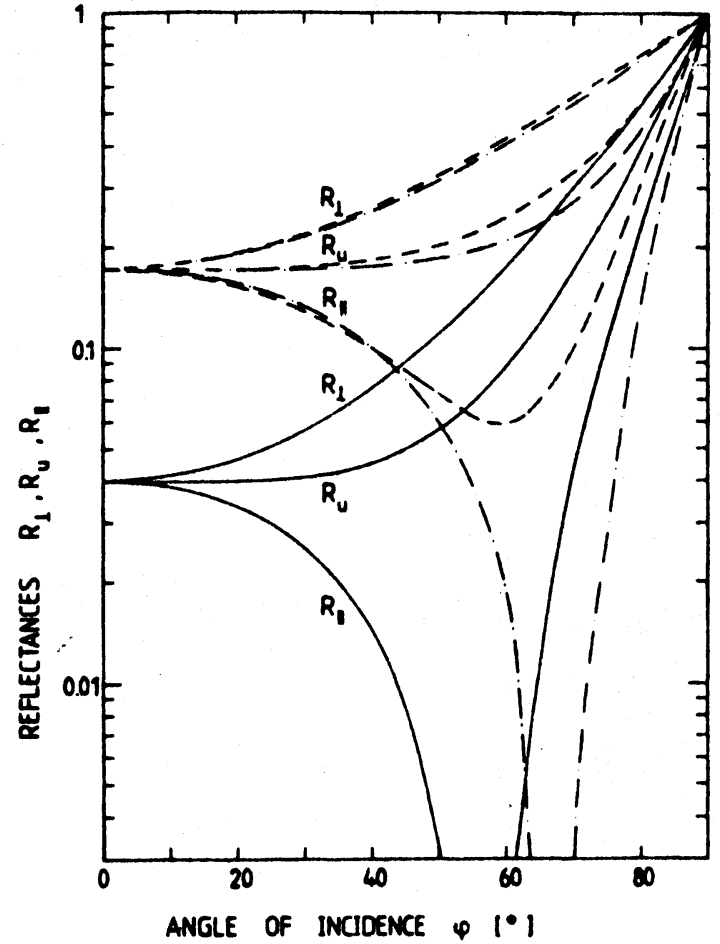

Fig. 2. Reflectance curves for perpendicular $\left(R_{\perp}\right)$, parallel $\left(R_{\mid}\right)$, and unpolarized $\left(R_{u}\right)$ incident light. The selected optical constants are (i) $n=1.5$ and $k=0$ (solid curves), (ii) $n=1.5$ and $k=1$ (dashed curves), and (iii) $n=2.42$ and $k=0$ (dash-dot curves).

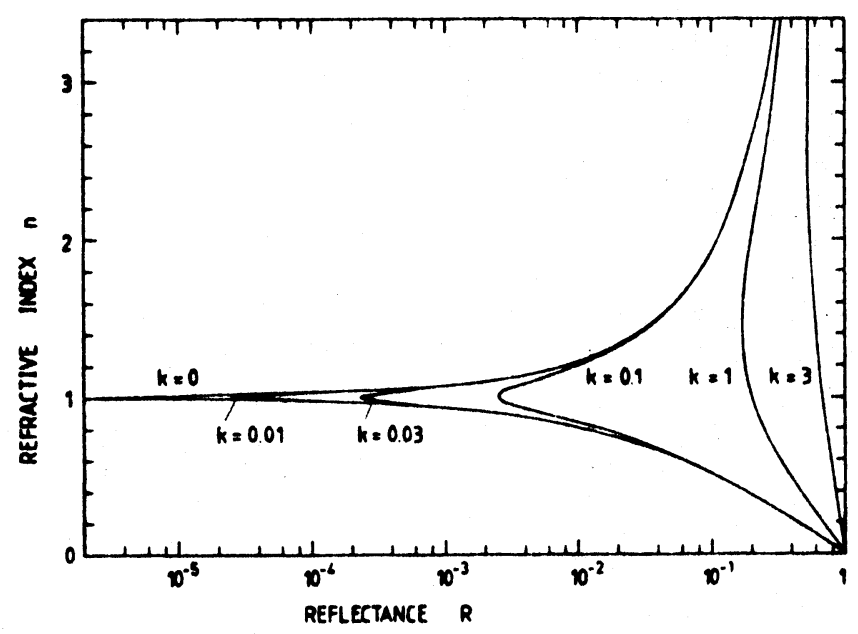

Fig. 3. Dependence of refractive index $n$ on reflectance $R$ for various extinction coefficients $k$. Angle of incidence is $\varphi=0^{\circ}$.

cating the difficulty and inaccuracy of simultaneous $n$ and $k$ determination from $R_{\perp}$ or $R_{u}$ measurements at two different angles of incidence. Between $60^{\circ}$ and $80^{\circ}$ the $R_{\|}$curves differ appreciably and allow simultaneous $n$ and $k$ determination. 30,31

Figure 3 gives a plot of $n$ vs $R$ for $\varphi=0^{\circ}$ and various extinction coefficients $k$. A low reflectance limit $R_{\min }$ exists for each $k$ which is found by setting the square root of Eq. (8) equal to zero. At $R>R_{\min }$ two solutions of $n$ exist. From dispersion theory the physically relevant solution has to be selected (in transparent regions the larger $n$ value is relevant). 


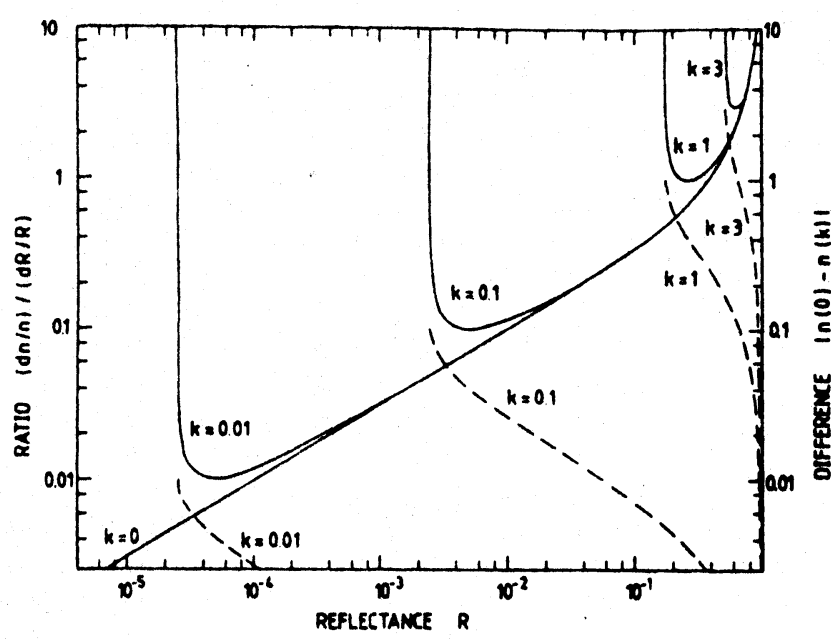

Fig. 4. Analysis of the sensitivity of refractive-index determination of reflectance measurement: solid curves, $(d n / n) /(d R / R)$ vs $R$; dashed curves, difference of refractive indices $|n(0)-n(k)|$ vs $R$. Curves are valid for angle of incidence $\varphi=0$ and for both branches of the $n(R)$ curves of Fig. 3 .

The accuracy of refractive-index determination of reflection measurement is revealed in Fig. 4 where $(d n / n) /(d R / R)=d \ln (n) / d \ln (R)$ vs $R$ is depicted $(\varphi=$ $0^{\circ}$, curves calculated from data of Fig. 3 ). At the positions $R_{\min }(k), d n / d R$ diverges [vertical tangent to $n(R)$ curve of Fig. 3], but $(\Delta n / n) /(\Delta R / R)$ remains finite for finite $\Delta R$. Reflection measurements with an accuracy of $\Delta R / R= \pm 0.002$ may be performed. ${ }^{32}$ For refractive indices near 1 (small reflectance) the accuracy of the refractive index by reflection measurement becomes rather high $\left[(d n / n) /(d R / R) \simeq R^{1 / 2}\right.$ for $\left.R<0.05\right]$. With increasing reflectance the accuracy lessens. At $R=0.04$ and $k \leq 0.1$ it is $n=1.5$ (Fig. 3) and $(d n / n) /(d R / R)=$ 0.2 (Fig. 4). With $\Delta R / R= \pm 0.002$ the uncertainty of the refractive-index measurement is $\Delta n / n= \pm 0.0004$ and $\Delta n= \pm 0.0006$. At a reflectivity of $R=0.3$ and $k$ $<1$ the uncertainty of measured refractive indices is already $\Delta n= \pm 0.007[n \simeq 3.5,(\Delta n / n) /(\Delta R / R) \simeq 1$, $\Delta R / R \simeq \pm 0.002]$.

With increasing reflectance $R$ the difference between $n(k)$ and $n(k=0)$ decreases as shown by the dashed curves in Fig. 4. For reflectances $R \gtrsim 0.02$ and extinction coefficients $k<0.01\left(\alpha<2500 \mathrm{~cm}^{-1}, \lambda=500 \mathrm{~nm}\right)$ the difference is $|n(k<0.01)-n(k=0)|<10^{-4}$.

\section{Experiment}

The experimental setup for the reflection measurements is shown in Fig. 5. A 200-W high-pressure mercury arc lamp or a $200-W$ halogen tungsten lamp is used as light source $L$. A parallel light beam is formed with slit $S 1$, aperture $A$, and lens $L 1$ (distance $S 1-L 1$ equal to focal length of $L 1$ ). Lens $L 3$ focuses the beam on the surface of the solid or liquid under investigation. Liquids are contained in open beakers and the light is directed nearly vertically $\left(\varphi=6.8^{\circ}\right)$ to the horizontal liquid surface (deflection prism P3). Polished solids are horizontally adjusted to the same position. The reflected light is deflected to the horizontal direction with prism $P 4$ and imaged to slit $S 2$ of the spectrograph

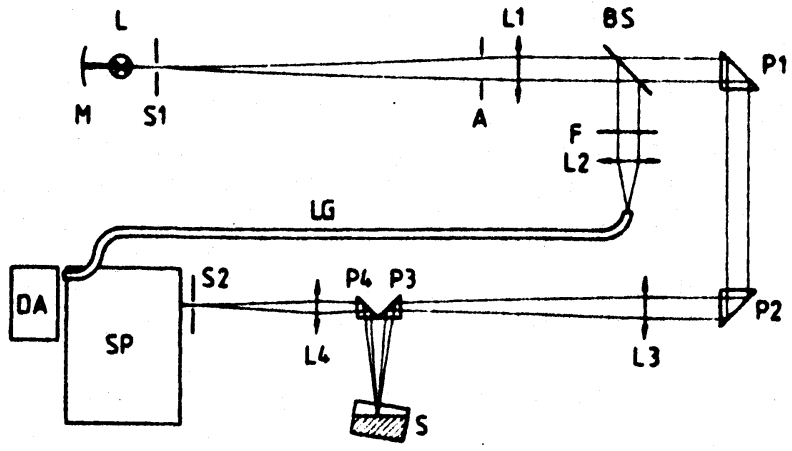

Fig. 5. Experimental setup: $L$, lamp; $M$, light collecting mirror; $S 1$ and $S 2$, slits (widths $S 1: 0.1 \mathrm{~mm}, S 2: 0.5 \mathrm{~mm}$ ); $A$, aperture (opening 1 cm); $L 1-L 4$, lenses (focal lengths $L 1: 1.33 \mathrm{~m}, L 2: 15 \mathrm{~cm}, L 3$ : $1 \mathrm{~m}, L 4: 5 \mathrm{~cm}$ ); $B S$, beam splitter; $F$, filters; $L G$, fiber-optic lightguide; $P 1-P 4,90^{\circ}$ deflection prisms; $S$, sample; $S P, 30-\mathrm{cm}$ spectrometer (grating with 1200 lines/mm); $D A$, silicon diode array (Tracor DARRS).

$S P$ with lens $L 4$. The 30 -cm grating spectrograph (1200 lines $/ \mathrm{mm}$ ) disperses the reflected spectrum to the silicon diode array detector DA (DARRS system of Tracor).

The spectral signal $S(\lambda)$ of the substance under in. vestigation is compared with the spectral signal $S_{\text {ref }}(\lambda)$ of a reference liquid of known reflectance $R_{u, r e f}(\lambda)$ [known refractive index $n_{\text {ref }}(\lambda)$ ]. Water was used as the reference in our case. The reflectance of the investigated substance is

$$
R_{u}(\lambda)=R_{u, \text { ref }}(\lambda) \frac{S(\lambda)}{S_{\text {rof }}(\lambda)}
$$

To get rid of fluctuations in the light source, a fraction of the light is separated with beam splitter $B S$, spectrally filtered $(F)$ and focused (lens $L 2$ ) to a fiber-optic lightguide and is detected at a side region of the diode array. The signals $S(\lambda)$ and $S_{\text {ref }}(\lambda)$ are normalized to the signal of the lightguide.

The width of slit $S 2$ was set to $d=0.5 \mathrm{~mm}$. The width of the light spot at slit $S 2$ was $\sim 0.1 \mathrm{~mm}$ limiting the spectral resolution to $\Delta \lambda \simeq 0.4 \mathrm{~nm}$. The obtained accuracy of the reflection measurements depended on the light source. Using the high-pressure mercury lamp the signals $S(\lambda)$ and $S_{\text {ref }}(\lambda)$ could be measured to an accuracy of $\Delta S / S \simeq \pm 0.002$ in regions of flat spectral emission while in regions of spectral lines the resolution reduced to $\Delta S / S \simeq \pm 0.01$. The corresponding accuracies of the reflectivities according to Eq. (9) are $\Delta R_{u} / R_{u} \simeq \pm 0.004$ and \pm 0.02 . For values of $n$ around 1.3 and $k<0.1$ the resulting uncertainty in the refractive indices is between $\Delta n= \pm 0.001$ and \pm 0.005 (see Fig. 4). With the halogen tungsten lamp the same accuracy as with the high-pressure mercury lamp in the flat spectral regions was achieved. The measurements described below were carried out with the mercury lamp in the $400 \leq \lambda<530-\mathrm{nm}$ region and with the halogen tungsten lamp in the $530 \leq \lambda<600 \mathrm{~nm}$ range.

The determination of the extinction coefficient $k$ is carried out with a spectrophotometer where the trans. mission $T(\lambda)$ is measured. Liquid cells with $l=10-\mu \mathrm{m}$ thickness are commercially available. Transmission 


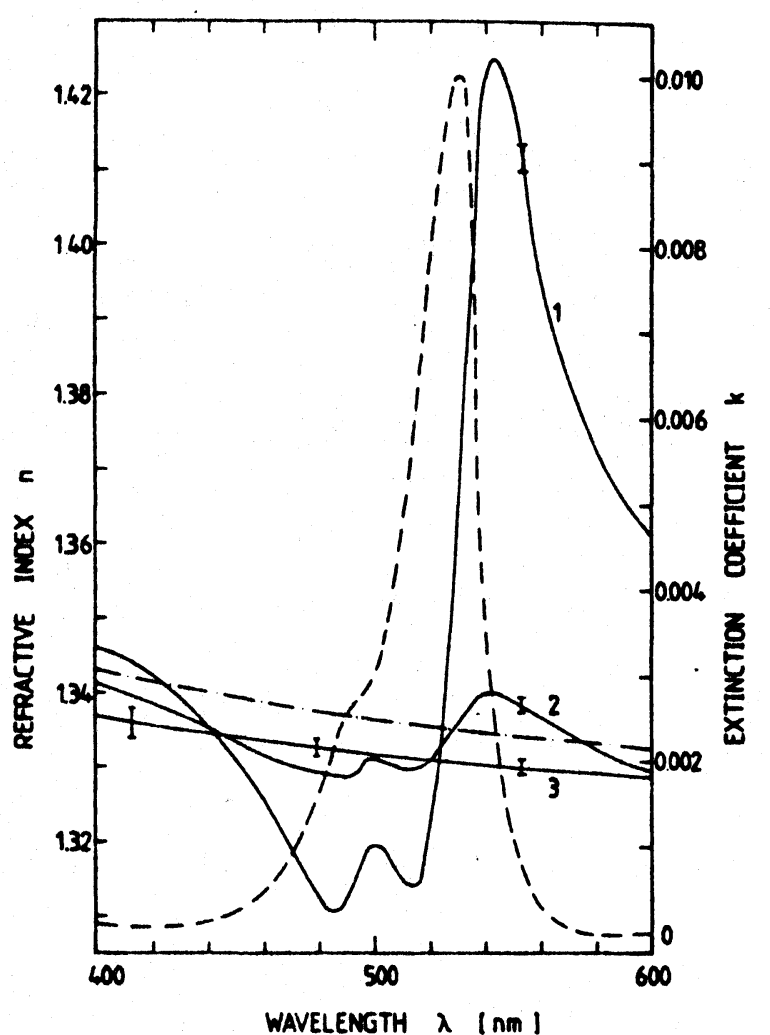

Fig. 6. Refractive indices and extinction coefficients vs wavelength. Temperature $20.2^{\circ} \mathrm{C}$. Solid curves, dispersion of refractive indices, (1) $0.1-\mathrm{M}$ rhodamine $6 \mathrm{G}$ in methanol, (2) $0.01-\mathrm{M}$ rhodamine $6 \mathrm{G}$ in methanol, (3) methanol. Dash-dot curve, dispersion of water (reference). Dashed curve, extinction coefficient of 0.01-M rhodamine $6 \mathrm{G}$ in methanol.

down to $T \simeq 10^{-3}$ is measurable. The corresponding maximum absorption coefficient and extinction coefficient are $\alpha_{\max }=-\ln (T) / l \simeq 7 \times 10^{3} \mathrm{~cm}^{-1}$ and $k_{\max }=$ $\alpha_{\max } /(4 \pi \tilde{\nu}) \simeq 3 \times 10^{-2}(\lambda=500 \mathrm{~nm})$, respectively.

\section{Results}

For illustration we present the optical dispersion of 0.01 - and $0.1-\mathrm{M}$ rhodamine $6 \mathrm{G}$ dissolved in methanol within the wavelength region from 400 to $600 \mathrm{~nm}\left(S_{0}-S_{1}\right.$ absorption peak at $530 \mathrm{~nm}$ ). Figure 6 depicts the measured refractive-index curves of the solvent methanol and of the rhodamine $6 \mathrm{G}$ solutions (temperature $20.2^{\circ} \mathrm{C}$ ). The refractive-index curve of the reference liquid water is included (data from Ref. 33). The methanol dispersion curve agrees within $\Delta n= \pm 10^{-3}$ with literature values. ${ }^{33}$ Within this accuracy there is no difference between the used surface reflection technique and prism techniques indicating no measurable influence of surface inhomogeneities or surface contamination.

The measured extinction coefficient $k$ of $0.01-\mathrm{M}$ rhodamine 6G solution is included in Fig. 6. The extinction coefficient of the $0.1-\mathrm{M}$ solution is assumed to be a factor of 10 larger (concentration independent constant absorption cross section). At this concentration a direct transmission measurement around the absorption peak is impossible since the absorption is too strong.
The wavelength dependence of the refractive indices of rhodamine 6G exhibits a superposition of two Lorentzian dispersion shapes resulting from the $S_{0}-S_{1}$ absorption peak at $530 \mathrm{~nm}$ and the vibronic $S_{0}-S_{1}$ absorption shoulder at $495 \mathrm{~nm}$. The refractive-index curves more clearly show up the vibronic $S_{0}-S_{1}$ transitions than does the absorption spectrum.

The refractive-index dispersion of dye solutions may be estimated from molecular dispersion theory. The relative permittivity is $c=n^{\prime 2}=1+\chi_{S}+\chi_{D}=n \xi+\chi_{D}$ $=n_{S}^{2}+\chi_{D}^{\prime}-i \chi_{D}^{\prime}\left(\chi_{S}\right.$ solvent susceptibility, $\chi_{D}$ dye susceptibility, $n_{S}$ refractive index of solvent). For $\chi_{D}$ $\ll n_{S}^{2}+\chi_{D}^{\prime}$ and $\chi_{D}^{\prime} \ll n_{S}^{2}$ the refractive index may be approximated by $n^{\prime}=\varepsilon^{1 / 2}=n-i k \approx\left(n_{S}^{2}+\chi_{\nu}^{\prime}\right)^{1 / 2}-$ $i \chi_{D} /\left[2\left(n_{S}^{2}+\chi_{D}\right)^{1 / 2}\right] \simeq\left(n_{S}^{2}+\chi_{D}^{\prime}\right)^{1 / 2}-i \chi_{D}^{\prime} /\left(2 n_{S}\right)$. From molecular dispersion theory ${ }^{34}$ for $m$ transitions follows $\chi_{D}=\sum_{j=1}^{m} A_{j}\left[\omega_{o j}^{2}-\omega^{2}+2 i \gamma_{j} \omega\right]^{-1}$, where $A_{j}$ is a consiant and $2 \gamma_{j}$ is the full width at half-maximum (FWHM) of the absorption band $j$. Rewriting in real and imaginary parts gives $\chi_{D}^{\prime \prime}=2 n_{S} k=2 n_{S} \sum_{j=1}^{m} k_{j \max }$ $4 \gamma_{j}^{2} \omega \omega_{o j}\left[\left(\omega_{o j}^{2}-\omega^{2}\right)^{2}+4 \gamma_{j}^{2} \omega^{2}\right]^{-1}$ and $\chi_{D}^{\prime}=2 n_{i} ; \sum_{j=1}^{m}$ $k_{j, \max }\left(\omega_{o j}^{2}-\omega^{2}\right) 2 \gamma_{j} \omega_{o j}\left[\left(\omega_{o j}^{2}-\omega^{2}\right)^{2}+4 \gamma_{j}^{2} \omega^{2}\right]^{-1}$. The peak extinction coefficient is $k_{j, \max }$ (at $\omega=\omega_{o j}$ ) of the $j$ th transition. Insertion of $\chi_{D}^{\prime}$ into $n$ gives $n=\left(n_{S}^{2}+\right.$ $\left.\chi_{D}^{\prime}\right)^{1 / 2} \simeq n_{S}+\chi_{D}^{\prime} /\left(2 n_{S}\right)=n_{S}+\sum_{j=1}^{m} k_{j, \max }\left(\omega_{o j}^{2}-\right.$ $\left.\omega^{2}\right) 2 \gamma_{j} \omega_{o j}\left[\left(\omega_{o j}^{2}-\omega^{2}\right)^{2}+4 \gamma_{j}^{2} \omega^{2}\right]-1$. For a single transition $(m=1)$ the refractive-index extrema are approximately given by $n\left(\omega_{01} \pm \gamma_{1}\right)=n_{S} \mp k_{1, \max } / 2$. In the case of our solutions of rhodamine $6 \mathrm{G}$ in methanol the single transition model gives $n(540 \mathrm{~nm})=n_{\max } \simeq$ $1.38(0.1 \mathrm{M})$ and $\simeq 1.337(0.01 \mathrm{M})$. The deviation from the experimental data is caused by contributions from other transitions.

\section{v. Conclusions}

The described technique of refractive-index detection by reflection measurement is practically not influenced by absorption up to $k \simeq 0.01$, the approximate limit measurable by transmission measurements. For typical refractive-index values around $n \simeq 1.5$ an accuracy of $\Delta n \simeq \pm 10^{-3}$ is achievable. The spectral range extends from 350 to $1100 \mathrm{~nm}$ for glass optics and is extendable to $220 \mathrm{~nm}$ with fused silica optics. The technique is applicable to strongly absorbing media $(k>0.03)$ if the extinction coefficient $k$ can be determined by some method other than transmission measurement (e.g., from the absorption behavior of diluted solutions).

\section{References}

1. H. Kessler, in Handbuch der Physik XVIII. Geometrischr Optik, optische Konstante, optische Instrumente, H. Geiger and K. Scheel, Eds. (Springer, Berlin, 1927), p. 623.

2. G. E. Fishter, in Applied Optics and Optical Engineering, Vol. 5, Part 1, R. Kingslake, Ed. (Acadernic, New York, 1967), p. 363

3. K. Schmid and A. Penzkofer, Appl. Opt. 22, 1824 (1983).

4. W. König, in Handbuch der Physik XX. Licht als Wellenbe. wegung, H. Geiger and K. Scheel, Eds. (Springer, Berlin, 1928), p. 242.

5. R. W. Ditchburn, Light (Academic, London, 1976), p. 534 
6. H. Gobrecht, in Bergmann.Schaefer Lehrbuch der Experimentalphysik, Band III Optik, H. Gobrecht, Ed. (Walter de Gruyter, Berlin, 1974), p. 265.

7. R. H. Muller, Surf. Sci. 16, 14 (1969).

8. R. M. A. Azzam and N. M. Bashara, Ellipsometry and Polarized Light (North-Holland, Amsterdam, 1977).

9. A. V. Sokolov, Optical Properties of Metals (American Elsevier, New York, 1967).

10. F. Abelés, Phys. Thin Films 6, 151 (1971).

11. F. Oswald and R. Schade, Z. Naturforsch. Teil A 9, 611 (1954).

12. T. E. Denton, R. D. Campbell, and S. G. Tomlin, J. Phys. D 5, 852 (1972).

13. J. R. Beattie and G. K. T. Conn, Philos. Mag. 46, 135 (1955).

14. O. Hunderi, Appl. Opt. 11, 1572 (1972).

15. V. M. Bermudez, Surf. Sci. 94, 29 (1980).

16. R. M. A. Azzam, J. Opt. Soc. Am. 72, 1439 (1982).

17. R. Tousey, J. Opt. Soc. Am. 29, 235 (1939).

18. I. Simon, J. Opt. Soc. Am. 41, 336 (1951).

19. D. G. Avery, Proc. Phys. Soc. London Sect. B 65, 425 (1952).

20. N. Neuroth, Z. Phys. 144, 85 (1956).

21. S. P. F. Humpreys-Owen, Proc. Phys. Soc. London 77, 949 (1961).

22. T. Hirschfeld, Appl. Spectrosc. 24, 277 (1970).

23. D. M. Kolb, J. Opt. Soc. Am. 62, 599 (1972).

24. A. B. Buckman and N. M. Bashara, Phys. Rev. 174, 719 (1968).

25. C. L. Nagendra and G. K. M. Thutupalli, J. Phys. D 15, 1153 (1982).

26. J. C. Phillips, Solid State Phys. 18, 56 (1966).

27. W. Leupacher and A. Penzkofer, to be submitted.

28. M. Thalhammer and A. Penzkofer, Appl. Phys. B 32, 137 (1983).

29. M. R. Query, J. Opt. Soc. Am. 59, 876 (1969)

30. W. R. Hunter, J. Opt. Soc. Am. 55, 1197 (1965)

31. H. B. Holl, J. Opt. Soc. Am. 57, 683 (1967).

32. H. E. Bennett and J. M. Bennett, Phys. Thin Films 4, 1 (1967).

33. Landolt-Börnstein Tables, Optische Konstanten, K. H. Hellwege and A. M. Hellwege, Eds. (Springer, Berlin, 1962), Part 8, pp. 5-565.

34. W. Demtröder, Laser Spectroscopy: Basic Concepts and Instrumentation (Springer, Berlin, 1981).

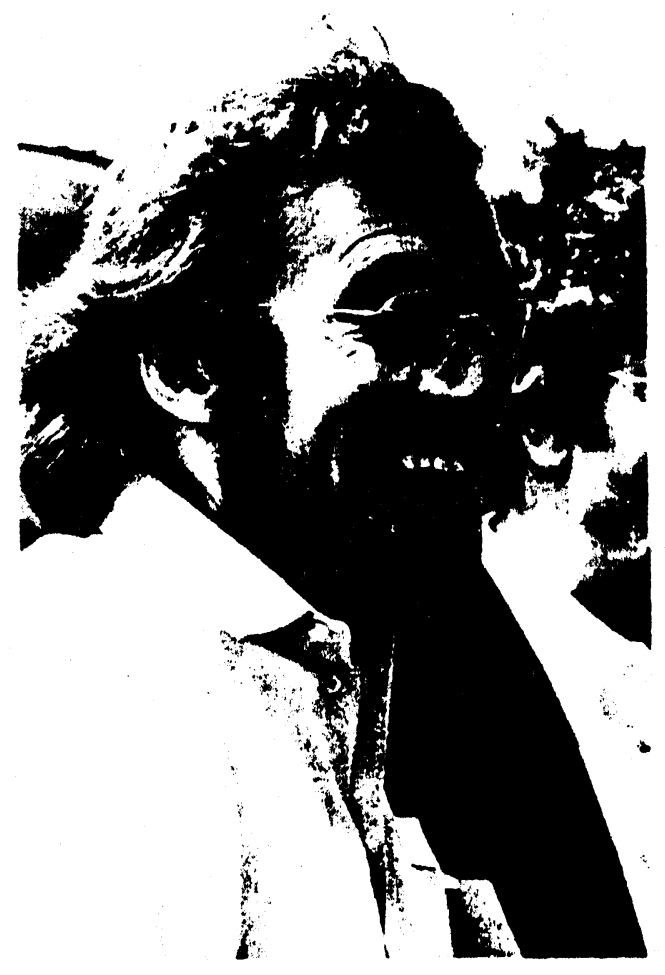

P. Meystre, of Max-Planck-Institut fur Quantenoptik, photographed by W. J. Tomlinson, of Bell Communications Research, during IQEC-12 in Munich, June 1982.

FIBER OPIICS

OPTICAL FIBER MEASUREMENT SYSTEYS AND METHODS

NBS fiber optics measurement systems and methods are described in detail in Optical Fiber Characterization-Vol. 2 (SP 637-2). The 239-page hardbound book covers measurements of attenuation, band width (frequency-domain), and far-field/near-field radiation pattern, plus a glossary of fiber optics tens and definitions. SP $637-2$ is available for $\$ 15$ prepaid from the National Bureau of Standards, Division 360.2, Boulder, Colo., 80303. It supplements an earlier publication (SP 637-1) on the measurement of optical fiber backscatter, time-domain bandwidth, and index profile, which is also available from the same address for $\$ 15$ prepaid. 